\title{
AN FPGA-BASED QUENCH DETECTION AND PROTECTION SYSTEM FOR SUPERCONDUCTING ACCELERATOR MAGNETS *
}

\author{
R. H. Carcagno ${ }^{\#}$, S. Feher, M. Lamm, A. Makulski, R. Nehring, D. F. Orris, Y. Pischalnikov, M. \\ Tartaglia, FNAL, Batavia, IL 60510, U.S.A.
}

\begin{abstract}
A new quench detection and protection system for superconducting accelerator magnets was developed for the Fermilab's Magnet Test Facility (MTF). This system is based on a Field-Programmable Gate Array (FPGA) module, and it is made of mostly commercially available, integrated hardware and software components. It provides all the functions of our existing VME-based quench detection and protection system, but in addition the new system is easily scalable to protect multiple magnets powered independently and provides a more powerful user interface and analysis tools. The new system has been used successfully for testing LHC Interaction Region Quadrupoles correctors and High Field Magnet HFDM04. In this paper we describe the system and present results.
\end{abstract}

\section{INTRODUCTION}

For more than eight years, Fermilab's MTF has been protecting superconducting magnets using a quench management system [1][2] based on NIM modules for Analog Quench Detection (AQD), a PowerPC running VxWorks for Digital Quench Detection (DQD) [3], and an in-house VME Quench Logic Module (QLM). This system has proven to be a very reliable solution, and there have been no incidents of superconducting magnet damage as a result of a malfunction of the quench management system.

As a result of a series of programs to test superconducting corrector coils (e.g., LHC Interaction Region Quadrupole correctors, Tevatron correctors, and the now defunct BTeV correctors), there was an interest to explore alternative quench management solutions that would be more cost effective, more portable, easier to configure and operate, and easier to scale up for the protection of multiple coils, each requiring multiple quench detection channels.

A solution was found based on a Field Programmable Gate Array (FPGA) module from National Instruments, in the rapidly growing PXI open standard components, and in Labview and Matlab software applications.

\section{THE FPGA MODULE}

The entire functionality of the MTF quench detection and protection standard has been implemented in the NI PXI-7831R FPGA module. With this FPGA module, the functionality of several NIM-based and VME-based modules has been consolidated into one small PXI component. This approach significantly reduces the cost and complexity of the quench detection and protection

*Work supported by the U.S. Department of Energy under contract No. DE-AC02-76CH03000.

"ruben $Q$ fnal.gov system without sacrificing functionality or performance. The main features of the NI FPGA module that were used in this application include the following: Eight simultaneous $16-\mathrm{bit}, 200 \mathrm{kHz}, \pm 10 \mathrm{~V}$ analog inputs; 11 TTL digital inputs; and 11 TTL digital outputs. The FPGA module runs the whole quench detection and protection logic in less than $5 \mu \mathrm{s}$, which is more than enough to protect any superconducting magnet that has been tested at MTF. Most of the time is spent in the Analog-to-digital conversion $(4 \mu \mathrm{s})$

\section{FPGA Module Functionality}

The following quench detection and protection functions have been implemented in the FPGA module:

- Six independent quench detection channels: (1) Whole Coil - Reference; (2) Whole Coil - Current Current Rate of Change; (3) Half Coil - Half Coil; (4) Cu Leads - Current; (5) SC Leads - Whole Coil; (6) Ground Current - Reference. For each channel, the user can specify threshold, balance, and offset. A balance mode with a current limit allows the user to disable quench detection channels for channel balancing purposes as long as the current does not exceed the specified limit.

- Four quench protection commands that are activated according to the logic of the quench detection channel that exceeded its threshold: (1) Zero Current; (2) Slow Current Ramp Down (3) Dump Fire; and (4) Heater Firing Unit (HFU) Fire. The user can specify a delay for Dump Fire and a delay for HFU Fire. In addition, a TTL signal and a PXI backplane trigger line are set in case of a quench detection channel trip to activate other quench management subsystems such as data logging for quench characterization.

- Up to three HFUs that can be configured in either protection mode or test mode. Only HFUs configured in protection mode respond to an $\mathrm{HFU}$ fire command. HFUs in test mode can be fired by the user at any time. For HFUs in protection mode, there is trip logic associated with their charge status and load connection status.

- First-Fault indication: in the event of a trip, this function indicates which fault condition was the first to cause the trip.

The functionality implemented in the FPGA module is sufficient for testing any superconducting magnet that MTF had to test over its eight years of operation.

\section{System Reliability}

Because the FPGA module is intended to protect expensive equipment, it is important to provide a system 
that is highly reliable and will fail in such a way that does not put the protected superconducting magnet at risk. The following features are included in the FPGA design to provide a highly reliable system:

- Heartbeat: the FPGA module generates a $10 \mathrm{KHz}$ TTL signal that is used by an independent electronics board to trip the system in the event that this heartbeat is lost.

- Normally closed (NC) logic: all signals must be high (TTL) or closed (contacts) to run. This logic prevents a failure to trip the system because of malfunctions such as a disconnected or broken cable, and will trip the system in case of loss of power to the PXI crate.

- Signal saturation protection: the FPGA logic monitors the proximity of analog signals to the input range limits $( \pm 10 \mathrm{~V})$, and in the event that a signal approaches these limits a slow ramp down is automatically initiated

- External Trip: an input is provided for backup or redundancy purposes, so independent equipment can be provided to detect a quench and issue a trip command.

The FPGA logic runs on firmware, completely independent of the PXI controller. It does not run under an operating system such as Windows, VxWorks, or Linux. Firmware logic execution is highly reliable. In this respect, the FPGA module is similar to the VME Quench Logic Module (QLM) that has been used at MTF for several years. Since the FPGA logic has been programmed in a high level language (Labview), it is very easy to understand what has been programmed. This would not have been the case if lower-level FPGA language such as VHDL was used. A simple, easy to understand firmware program is very important to assure the system will perform as expected and to make maintenance and upgrades easy.

\section{ADDITIONAL COMPONENTS}

In addition to the FPGA module, there are several components that have been included to provide a complete quench management system.

\section{The FPGA Graphical User Interface (GUI)}

The FPGA GUI is provided by a Labview 7.1 application running on a PXI-8186 controller. The FPGA module communicates with this controller via the PXI crate backplane. The PXI controller runs Windows XP, although it can also run a real-time operating system instead. Since the FPGA module provides all of the required real-time functionality, we chose to run Windows $\mathrm{XP}$ in the controller. This GUI is a key element that makes the new quench detection and protection system user friendly. The GUI provides easy access to all userspecified parameters, and provides monitoring of all FPGA analog and discrete values with a refresh rate of up to $10 \mathrm{~Hz}$. It also provides means to store and retrieve configuration information and visualize streaming analog data with charts. The user can run this GUI remotely by using the standard Windows XP Remote Desktop, or from any Web browser by using Labview's Remote Panels.

\section{Data Logging}

In the event of a trip or quench the system includes a set of 8-channel, 16 bit, simultaneously sampled PXI6143 data loggers for quench characterization purposes. The user can configure the sample rate, number of samples, and number of pre-trigger samples for these dataloggers. Sample rates can be as high as $250 \mathrm{Ks} / \mathrm{s}$ per channel. Data loggers are triggered via the PXI backplane trigger lines, which are set by the FPGA module. A Labview 7.1 application is included to allow visualization of the logged signals with simple charts.

\section{Quench Analysis}

A powerful stand-alone Matlab application was developed for quench analysis purposes. Written in Matlab version 7.04 and compiled into a standalone application, the Quench Data Analysis (QDA) program, which is an interactive GUI, provides the user with many specialized functions for plotting and analyzing magnet quench signals. Some of its key features include:

- Digital Filtering (DSP) Tools

- Capability to easily switch between Time Domain and Frequency Domain plotting over an interactively user specified region

- Exponential fitting tool

- Quick signal rescaling of over-plotted data

- Data saving with user specified decimation rates.

- ASCII output files are compatible with Matlab and MS Excel, etc.

- Automatic report generation

The FPGA-based quench manager writes a binary file composed of multidimensional arrays that contain both raw data and quench parameter information. Upon reading this file the QDA program displays a list of quench signals, the quench summary information and quench testing parameters, and then plots the signal that caught the quench. The above-mentioned tools can then be used to quickly provide more detailed information about the quench event. For instance, the user can interactively perform an exponential fit over a specified region, such as the current decay, or switch from the time domain to the frequency domain over a user specified region. Information such as the resolution in time and frequency are automatically displayed, and a test report that includes magnet quench summary information along with several primary plots can be generated. In addition, user selected data can be decimated and written to a compact ASCII file for further analysis with Matlab or MS Excel. There are also several graphics tools and saveable user configuration preferences that help make analysis of the quench data more efficient.

\section{Power Supply Controller}

The system includes an 8 -channel, 16 bit, $1 \mathrm{MS} / \mathrm{s}$ analog output module PXI-6733 that can be used to provide the drive signal to several power systems concurrently. The 
GUI for the power supply controller is a Labview 7.1 application running in the PXI controller. This GUI allows the user to define and store ramp profiles and to start and stop ramps. During ramping, the GUI includes trend charts for the drive signal and current. In addition, there are several interlock conditions to coordinate the power supply controller with FPGA module logic and quench characterization logic. These interlocks make sure the user is not allowed to ramp a supply until all other systems are properly functioning and ready.

\section{Multiple Systems}

The system is designed in such a way that coexistence of multiple independent quench management systems in the same PXI crate is possible. For example, this system has been used to simultaneously power and protect Tevatron corrector coil packages, which include three separate superconducting coils. Three FPGA modules with their corresponding data loggers were installed in the same PXI crate, along with the power supply controller and the PXI controller running all GUIs. Navigation features make it easy to switch and recognize GUIs for different systems. Each FPGA module triggers a different PXI trigger line so the appropriate datalogger is triggered in case of a trip. A coil first-fault logic indicator is included in the FPGA logic to capture which coil quenched first in a sequence of coil quenches.

\section{APPLICATION EXAMPLES}

The FPGA-based Quench Management System has been successfully implemented and tested in two superconducting magnet test systems: One, a corrector coil measurement system; and two, a Vertical Magnet Test Facility (VMTF) [4] used to test high field superconducting magnets up to $30 \mathrm{KAmps}$ DC.

\section{Corrector Coil Tests}

Two tests were successfully performed using the corrector coil measurement system. The first was a test up to $500 \mathrm{Amps}$ on a variety of superconducting corrector coils with inductances up to $300 \mathrm{mH}$. Each corrector coil was powered tested separately. The second test was performed at lower currents, $\sim 75 \mathrm{Amps}$. However, for this test up to three corrector coils were powered simultaneously to verify that the quench performance was not negatively affected by the coupling of the coils. For this test, the multiple systems configuration described above was used.

\section{High Field Magnet Test}

The high field magnet HFDM04 was successfully quench tested using the new FPGA-based quench management system in VMTF. For this magnet, quench propagation heaters as well as an energy extraction (dump) resistor were required in order to protect the magnet conductor in the event of a quench. Parameters such as heater delay and dump delay were input by the user; the quench manager carried out the necessary quench logic. Figure 1 shows a quench at 6765 Amps.

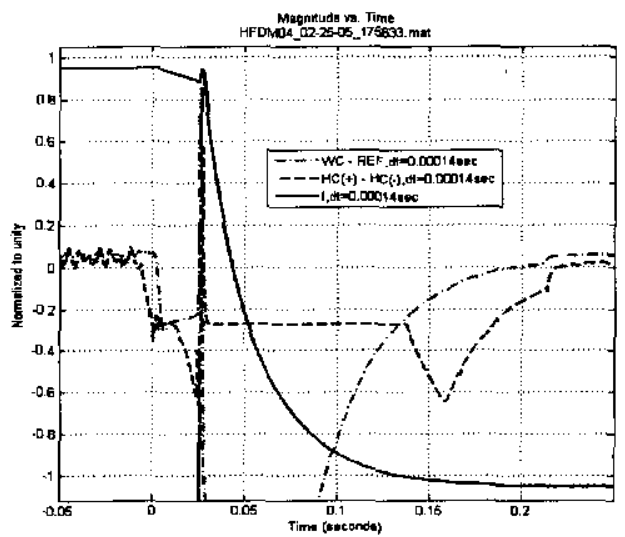

Figure 1: Quench Detection signals with rescaled Whole Coil, Bucked Half Coils, and Current. Quench current is 6765Amps.

Note that three quench signals have been over-plotted and rescaled to compare over a wide dynamic range. It can be seen from the bucked half coils signal that the quench developed in $7 \mathrm{~ms}$ before time $t=0$. At $t=0$ the power supply current (I) is ramped down. At the user specified dump delay of $25 \mathrm{~ms}$ the energy extraction resistor is switched into the circuit causing the current to decay much faster and a sharp spike to be generated on the coil voltage signals. Since the quench detection amplifiers are set to a higher gain in order to improve sensitivity, the bucked half coils signal quickly becomes unbalanced after the quench and saturates. However, the Quench Manager also triggers data loggers whose input amplifiers are set to a lower gain in order to capture the entire signal both before and after the quench.

In summary, the FPGA-based quench manager provides the same level of quench protection logic as the VME based system with less hardware and yet is more flexible.

\section{REFERENCES}

[1] D.F. Orris, R. Carcagno, S. Feher, M.J. Lamm, J. Nogiec, S. Sharonov, P. Schlabach, M. Tartaglia, and J. Tompkins, "A Quench Management System for Testing Superconducting Magnets", ASC2002, Houston, Texas, (2002)

[2] R. Carcagno, D.F. Orris, "A Modular and Extensible Data Acquisition and Control System for Testing Superconducting Magnets," Particle Accelerator Conference (PAC), 2001.

[3] D.F. Orris, S. Feher, M.J. Lamm, J. Nogiec, S. Sharonov, M. Tartaglia, and J. Tompkins, "Digital Quench Detection System for Superconducting Magnets", PAC'99, New York, (1999)

[4] M.J. Lamm et al., "A New Facility to test Superconducting Accelerator Magnets", PAC'97, Vancouver, Canada, 1997 